\title{
Beneficios de utilizar el Cuestionario para Evaluar las Disfuncionalidades de las Articulaciones Temporomandibulares en Artritis Reumatoide (CEDATAR)
}

\section{Benefits of using the Questionnaire to Assess Temporomandibular Joint Dysfunctionalities in Rheumatoid Arthritis (CEDATAR)}

DOI: 10.46981/sfjhv2n4-009

Received in: Oct 1st, 2021

Accepted in: Dec 31th, 2021

\author{
Karen Vanesa Rhys \\ Mg. Formador de Formadores \\ Universidad Nacional de Córdoba \\ E-mail: rhyskaren@gmail.com \\ Carla Andrea Gobbi \\ Dra. en Medicina \\ Universidad Nacional de Córdoba \\ E-mail: carlaandreagobbi@hotmail.com \\ Beatriz Busamia \\ Dra. en Odontología \\ Universidad Nacional de Córdoba \\ E-mail: beabusamia@gmail.com \\ Eduardo Albiero \\ Dr. en Medicina \\ Universidad Nacional de Córdoba \\ E-mail: eduardoalbiero@gmail.com \\ Ana Laura Lagnarini \\ Dra. en Odontología \\ Universidad Nacional de Córdoba \\ E-mail: lauralagnarini@gmail.com \\ Paula Alba \\ Dra. en Medicina \\ Universidad Nacional de Córdoba \\ E-mail: paulaalba@yahoo.com
}

\section{RESUMEN}

Han aumentado las técnicas para la examinación clínica e imagenológica de las articulaciones temporomandibulares, sin embargo, no sucede lo mismo en la posibilidad de evaluación en la anamnesis cotidiana en la consulta de rutina. Existen muy pocos cuestionarios y la mayoría son antiguos. Objetivo: Utilizar una herramienta útil y práctica para las interconsultas y derivaciones de pacientes entre el Servicio de Reumatología y el Servicio de Odontología. Material y métodos: Se implementó el Cuestionario para evaluar las disfuncionalidades de las articulaciones temporomandibulares en artritis reumatoide (CEDATAR) para evaluar si presentaba la capacidad de detectar alteraciones en las 
articulaciones que le sirvieran a los distintos profesionales en la decisión de derivar a un especialista al paciente. Resultados: Se evidenció que todos los pacientes que acusaron alteraciones en las respuestas del cuestionario, al corroborarse presentaron modificaciones importantes en el análisis imagenológico y/o clínico. Conclusiones: La artritis reumatoide es una enfermedad declarada de importancia en salud pública y políticas sanitarias, es de imponderable relevancia la utilización de CEDATAR en las consultas reumatológicas cotidianas para agilizar las terapéuticas limitando el avance de la enfermedad autoinmune a nivel de las articulaciones temporomandibulares suministrando un pronóstico más favorable y limitando lesiones y discapacidades.

Palabras claves: Cuestionario, Articulación temporomandibular, ATM, Beneficios, Artritis reumatoide.

\section{ABSTRACT}

Techniques for clinical and imaging examination of the temporomandibular joints have increased, however, the same does not happen in the possibility of evaluation in the daily anamnesis in the routine consultation. There are very few questionnaires and most are old. Objective: To use a useful and practical tool for interconsultations and referrals of patients between the Rheumatology Service and the Dentistry Service. Material and methods: The Questionnaire to evaluate temporomandibular joint dysfunctionalities in rheumatoid arthritis (CEDATAR) was implemented to assess whether it had the ability to detect joint alterations that would help the different professionals in the decision to refer a specialist to the patient. Results: It was evidenced that all the patients who reported alterations in the responses to the questionnaire, when corroborated, presented important modifications in the imaging and / or clinical analysis. Conclusions: Rheumatoid arthritis is a disease declared of importance in public health and health policies, the use of CEDATAR in daily rheumatology consultations is of imponderable relevance to expedite therapeutics by limiting the advance of autoimmune disease at the level of the temporomandibular joints, providing a more favorable prognosis and limiting injuries and disabilities.

Keywords: Questionnaire, Temporomandibular joint, TMJ, Benefits, Rheumatoid arthritis.

\section{INTRODUCCIÓN}

Las articulaciones temporomandibulares son las de mayor actividad, ya que intervienen en la alimentación y la fonación entre otras praxias. Son las articulaciones más sociales. Razones por las que se debieran priorizar en la atención estomatognática del paciente. Aunque las anomalías estructurales de las ATM pueden predisponer a síntomas, se debe reconocer que no todos sufren el mismo nivel de sintomatología ${ }^{1}$. Estas variaciones en los signos y síntomas llevan a que se debe afinar e insistir en un accionar temprano para la detección precoz de alteraciones. Se suma que los pacientes con enfermedades autoinmunes tienen particularidades especiales, a la vez que son más vulnerables y se deberían considerar con mayor reparo ${ }^{2}$. Estas son causas que justifican el probar cuestionarios actualizados para facilitar la tarea profesional y el diagnóstico y tratamiento precoz de los pacientes.

La artritis reumatoide es una enfermedad autoinmune sistémica degenerativa inflamatoria crónica y progresiva que afecta las articulaciones. Entre las articulaciones comprometidas la Sociedad Argentina de Reumatología cita: “inflamación articular en forma simétrica de pequeñas y grandes articulaciones como manos, pies, rodillas, codos, hombros, caderas, pudiendo comprometer 
articulaciones diartrodiales como las témporomandibulares y cricoaritenoideas". Actualmente los criterios de diagnóstico se basan en los publicados por la American College of Rheumatology . $^{3,4}$.

\section{OBJETIVOS}

- $\quad$ Utilizar una herramienta útil y práctica para las interconsultas y derivaciones de pacientes entre el Servicio de Reumatología y el Servicio de Odontología.

\section{MATERIAL Y MÉTODOS}

Estudio observacional, descriptivo, prospectivo y de corte transversal, establecido en serie de casos. Se utilizó el: Cuestionario para evaluar las disfuncionalidades de las articulaciones temporomandibulares en artritis reumatoide (CEDATAR $)^{5}$. Es un cuestionario dicotómico, de cuatro dimensiones: dinámica, estática, traslación en cierre y traslación en apertura para que realice el médico reumatólogo y en base a los resultados conozca la necesidad o no de derivar al profesional odontólogo.

\begin{tabular}{|c|c|c|}
\hline & $\mathrm{Si}$ & No \\
\hline Al bostezar, ¿siente dolor en la cara del lado derecho? & & \\
\hline Al bostezar, ¿siente dolor en la cara del lado izquierdo? & & \\
\hline Al bostezar, ¿siente dolor en el cuello del lado derecho? & & \\
\hline Al bostezar, ¿siente dolor en el cuello del lado izquierdo? & & \\
\hline ¿Siente ruidos del lado derecho al abrir la boca? & & \\
\hline ¿Siente ruidos del lado izquierdo al abrir la boca? & & \\
\hline ¿Siente ruidos del lado derecho al cerrar la boca? & & \\
\hline ¿Siente ruidos del lado izquierdo al cerrar la boca? & & \\
\hline ¿Siente zumbidos en el oído derecho? & & \\
\hline ¿Siente zumbidos en el oído izquierdo? & & \\
\hline ¿Siente dolores de cabeza a los costados? & & \\
\hline $\begin{array}{l}\text { 12. ¿Puede morder alimentos duros o semiduros como nueces o tostadas a la } \\
\text { mañana? }\end{array}$ & & \\
\hline $\begin{array}{l}13 . \\
\text { tarde? }\end{array}$ ¿Puede morder alimentos duros o semiduros como nueces o tostadas a la & & \\
\hline 14. ¿Puede masticar del lado derecho igual que del lado izquierdo? & & \\
\hline $\begin{array}{l}\text { 15. ¿Siente que cuando mastica por más de dos minutos seguidos se cansa y se } \\
\text { le dificulta continuar masticando? }\end{array}$ & & \\
\hline Al mover la mandíbula hacia la derecha, ¿siente molestias? & & \\
\hline Al mover la mandíbula hacia la izquierda, ¿siente molestias? & & \\
\hline Al adelantar la mandíbula, ¿siente molestia? & & \\
\hline $\begin{array}{l}\text { 19. Cuando pasa un tiempo largo sin mover la boca, ni comer, ni hablar, ¿siente } \\
\text { molestia? }\end{array}$ & & \\
\hline 20. ¿Siente dolor si aprieta fuerte las muelas mordiendo unas contra otras? & & \\
\hline
\end{tabular}

Se realizó a cien pacientes que asistieron al Servicio de Reumatología del Hospital Córdoba. En forma aleatoria a algunos de estos pacientes se les realizaron ecografías de alta resolución power Doppler, en las articulaciones temporomandibulares para determinar la existencia o no de patología, a la vez que se efectuaron imágenes ecográficas de las articulaciones de las manos para corroborar y 
comparar el avance de la enfermedad. A un pequeño porcentaje se le indicaron resonancias magnéticas de las articulaciones temporomandibulares ${ }^{8,9}$. Los pacientes debieron firmar el consentimiento informado y presentar un diagnóstico de artritis reumatoide según los criterios 2010 para AR de American College of Rheumatology. El presente estudio se encuentra aprobado por el Cieis del Adulto del Hospital Córdoba.

\section{RESULTADOS}

Ningún paciente expresó per se, a los profesionales reumatólogos sintomatología referida a las articulaciones temporomandibulares. Otros pacientes consideraron que si no existe dolor en las articulaciones temporomandibulares no es necesario informar al médico o al odontólogo. A estas dos situaciones desfavorables se suma que las personas con artritis reumatoide se encuentran polimedicados y esto solapa el avance de la enfermedad en estas zonas en particular. Sin embargo, al realizar CEDATAR se demostró que relataban por el simple hecho de responder a la pregunta síntomas que afectaban las articulaciones. Para evaluar las articulaciones en forma objetiva y corroboran se realizaron ecografías y resonancias magnéticas.

Se hallaron irregularidades y erosiones óseas en las articulaciones temporomandibulares y se compararon en porcentaje con las manos como articulaciones de referencia.

Irregularidades y erosiones óseas de ATM

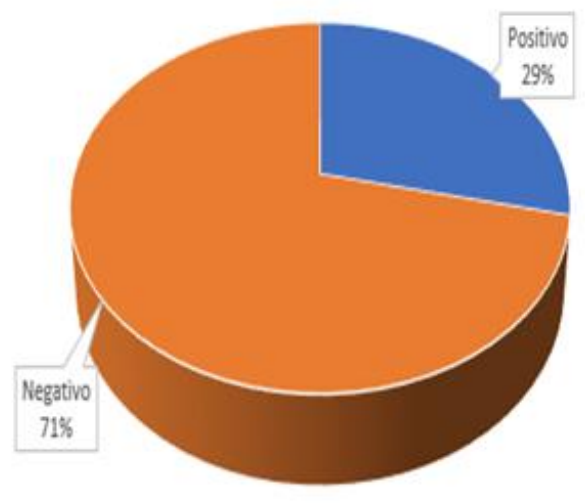

Irregularidades y erosiones óseas de manos

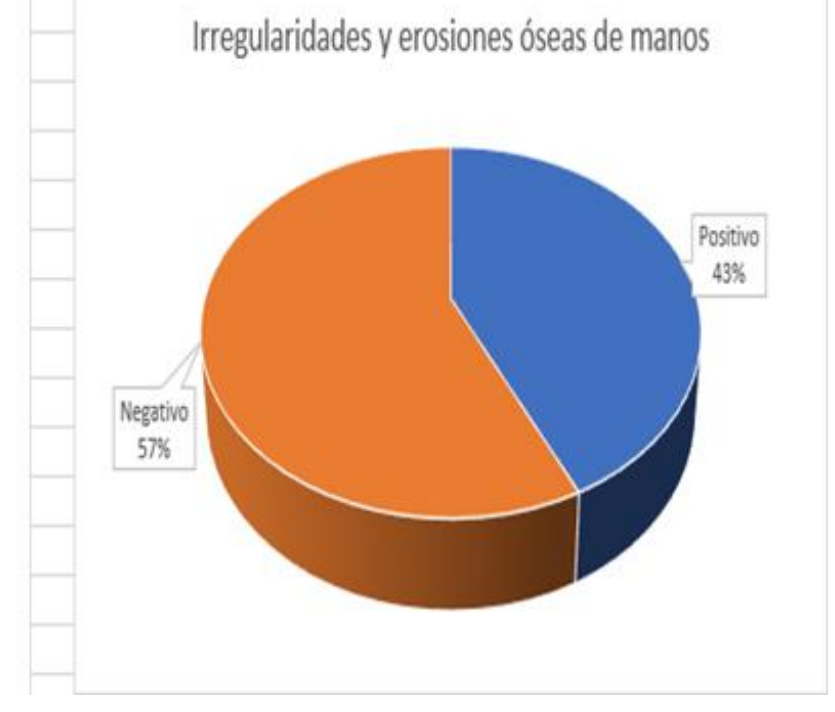

Gráfico que muestra la presencia de irregularidades y erosiones óseas en pacientes que completaron positivamente Cedatar.

Se observó que el $43 \%$ de los pacientes evidenciaba derrame articular. Un $2 \%$ presentaba distensión capsular. 


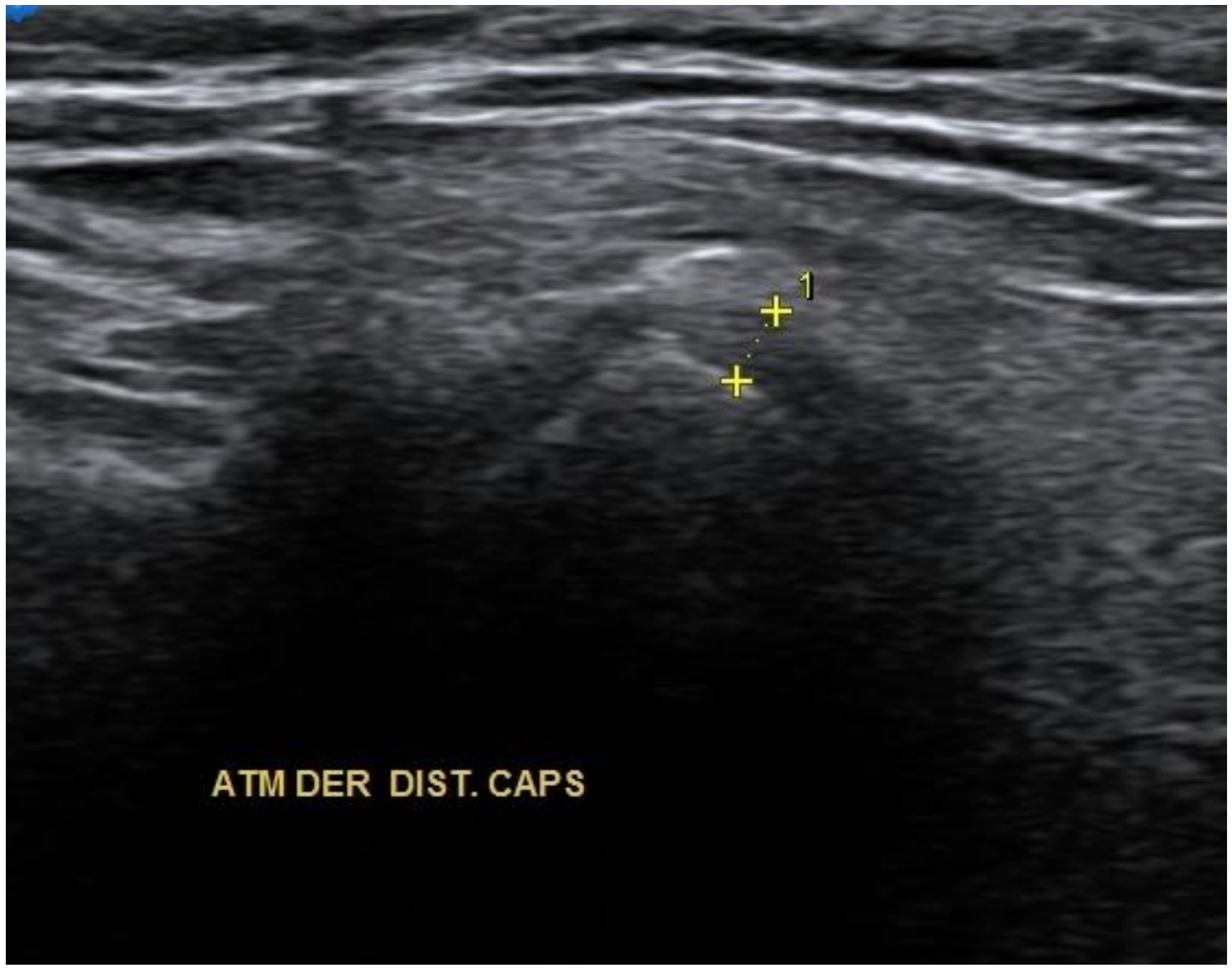

Ecografía de la articulación temporomandibular derecha que muestra derrame articular y distensión capsular

El $73 \%$ de los pacientes consideraba que "era normal, por su enfermedad" el presentar limitaciones en las articulaciones y que ya no tenían solución terapéutica. El 56\% relató que aumentaban la dosis de antiinflamatorios en los días que las sentían afectadas o trabadas porque desconocían la existencia de tratamientos específicos para estas articulaciones en particular.

\section{CONCLUSIONES}

Completar CEDATAR en las consultas permite que el paciente tome conciencia de que la artritis reumatoide puede afectar sus articulaciones temporomandibulares y consulte a otros profesionales de la salud como los odontólogos.

Ya que la artritis reumatoide es una enfermedad declarada de importancia en la salud pública y las políticas sanitarias, es de imponderable relevancia la utilización de CEDATAR en las consultas reumatológicas cotidianas para agilizar las terapéuticas limitando el avance de la enfermedad autoinmune a nivel de las articulaciones temporomandibulares suministrando un pronóstico más favorable y limitando lesiones y discapacidades. 


\section{BIBLIOGRAFÍA}

1- $\quad$ Li DTS, Leung YY. Trastornos temporomandibulares: conceptos actuales y controversias en diagnóstico y manejo. Diagnóstico (Basilea). 6 de marzo de 2021; 11 (3): 459. doi: 10.3390 / diagnostics11030459. PMID: 33800948; PMCID: PMC8000442.

2- $\quad$ Rhys K, Guerrero C, Olmos J, Molina J, Pelliccioni P. Relación profesional de la Salud- Paciente. South Florida Journal of Development, Miami, v. 2, n. 3, edición especial, jul. 2021. DOI: https://doi.org/10.46932/sfjdv2n3-012

3- $\quad$ Scott DL, Wolfe F, Huizinga TW. Rheumatoid arthritis. Lancet. 376. 2010, pp. 1094-1108.

4- $\quad$ Aletaha D, Neogi T, Funovits AJ, Felson DT, Bingham CO. 3rd 2010 rheumatoid arthritis classification criteria: an American College of Rheumatology European League Against Rheumatism collaborative initiative. Ann rheum Dis., 69 2010, pp. 15801588.

5- $\quad$ Rhys K, Gobbi C, Busamia B, Albiero E, Alba P, Lagnarini L. Validación del cuestionario: CEDATAR (cuestionario para evaluar las disfuncionalidades de las articulaciones temporomandibulares en artritis reumatoide). Revista Ocronos, ISSN: 2603-8358 - depósito legal CA27-2019. Vol. IV. Núm. 7 (Julio 2021) - Pág. Inicial: 66. https://revistamedica.com/validacioncuestionario-cedatar.

6- http://reumatologia.org.ar/normativa_cert_disc_pacientes_ar.php. 2017.

7- Revista Argentina de Reumatología. Realidad de la artritis reumatoidea en Argentina. ISSN 0327-4411. 2008. Año 19. N³. Pag. 10.

8- $\quad$ M.S. Saltzherr, J. H. Coert, R. W. Selles, J. W. van Neck, J.Bart Jaquet, G. J. V. M. van Osch, E. H. G. Oei, J. J. Luime, G. S. R. Muradin. Accuracy of magnetic resonance imaging to detect cartilage loss in severe osteoarthritis of the first carpometacarpal joint: comparison with histological evaluation. Arthritis Res Ther. 2017; 19: 55. Published online 2017 Mar 14. doi: 10.1186/s13075-017-1262-8. PMCID: PMC5348904.

9- Manoliu A, Spinner G, Wyss M, Erni S, Ettlin D.A., Nanz D, Ulbrich E.J., Gallo L.M., Andreisek G. Comparación cuantitativa y cualitativa de la resonancia magnética de la articulación temporomandibular a 1,5 y 3,0 T utilizando un protocolo optimizado de alta resolución. Dentomaxillofac Radiol. 2016; 45 (1): 20150240. doi: 10.1259 / dmfr.20150240. Epub 201515 de septiembre. 